\title{
Enzyme-Histochemical Demonstration of the Intramural Lymphatic Network in the Monkey Jejunum
}

\author{
Hiroshi Shimoda $^{1}$, Seiji Kato ${ }^{2}$ and Tetsuji Kudo ${ }^{1}$ \\ Departments of Anatomy ${ }^{1}$ and Human Biology², Oita Medical University, Oita, Japan
}

Received February 17, 1997

\begin{abstract}
Summary. The structural organization of the intramural lymphatic networks in the jejunum of the Japanese monkey (Macaca fuscata) was studied by an enzymehistochemical method using whole-mount preparations of tissue layers. A distinction between the lymphatics and the blood vessels was made by light microscopy using $5^{\prime}$-nucleotidase (5'-Nase)-alkaline phosphatase (ALPase) double staining. In each tissue specimen, the reaction product for $5^{\prime}$-Nase activity was localized in the lymphatic vessels, whereas that for ALPase activity was restricted to blood vessels. The mucosal layer exhibited an irregular network of tubular lymphatics connecting to the central lymphatics in the villi. The submucosal layer revealed a horizontal mesh-like network consisting of tubulo-saccular lymphatics. A dense maze-like network of lymphatics was distributed throughout the myenteric layer. The lymphatic networks were independent of the blood vessels. The present results indicate the usefulness of the enzymehistochemistry of whole-mount preparations of the laminar layers for demonstration of the structure and distribution of the lymphatic system in the intestinal wall.
\end{abstract}

The lymphatic system of the gastrointestinal tract plays an important role in the transport of nutrient fluids such as the rich emulsion of fat, tissue fluid and cellular elements, both to the lymph nodes and to the blood stream (ALLEN, 1967). Accordingly, to examine microcirculatory disorders and metastases of malignant neoplasms to lymph nodes, the precise distribution of lymphatics must be clarified. While the structural organization of the lymphatic network in the gastrointestinal tract has been studied in several species by various methods including the injection of dyes or silver nitrate into the parenchyma or blood vessel, the differentiation of initial lymphatics from blood capillaries by light microscopic examination often proves difficult (VAJDA and TÖMBÖL, 1964; Mori, 1969; ZWEIFACH, 1973; SATOMURA et al., 1978).
Scanning electron microscopic studies of corrosion casts and of tissues after $\mathrm{KOH}$-collagenase treatment have revealed the three-dimensional organization of the lymphatics in the stomach and intestine of the rat, rabbit and canine (OHTANI and OHTSUKA, 1985; OHTANi, 1987, 1992; OHTANi and MURAKAMI, 1987; USHIKI, 1990; YAMANAKA et al., 1995; SUGiTo et al., 1996). However, the precise structure and distribution of the intramural initial lymphatic network in the gut have not been sufficiently demonstrated because of difficulties in identifying initial lymphatics by previously available methods.

Recently we developed an enzyme-histochemical method with $5^{\prime}$-nucleotidase ( $5^{\prime}$-Nase)-alkaline phosphatase (ALPase) double staining to distinguish lymphatics and blood capillaries on various tissue sections and whole-mount preparations of flat membranous organs such as the mesentery of various mammals, including humans (KATO and MIYAUCHI, 1989; KATO, 1990a, b; KATO and GoTOH, 1990; YASUNAGA et al., 1991a, b; KATO et al., 1991, 1993). In the present study, we applied our staining method to separated laminar layers (whole-mount preparations) of the jejunal wall to demonstrate the fine distribution of the lymphatic network. The intestine of a Japanese monkey, Macaca fuscata, was used in this study because of the absence of studies on the intestinal lymphatic system of primates, other than our previous reports (YASUNAGA et al., 1991a, b; KATO et al., 1991, 1993).

\section{MATERIALS AND METHODS}

Twelve young Japanese monkeys (Macaca fuscata) of both sexes, weighing $3-5 \mathrm{~kg}$, were used. They were kept under standard laboratory conditions, and all 
experiments were carried out according to the Guidelines for Animal Experimentation, Oita Medical University.

\section{Tissue preparation}

After the animals were euthanized by exsanguination under deep anesthesia with sodium pentobarbital ( 25 $\mathrm{mg} / \mathrm{kg}$ ), the jejunal segments were removed and placed in $0.1 \mathrm{M}$ cacodylate buffer ( $\mathrm{pH} 7.2$ ) containing $7 \%$ sucrose. These intestinal segments were cut along the mesenteric border, and intestinal contents were flushed away with the buffer described above. The tissues were then stretched, pinned on a plastic board with the mucosal side up, and immersed in a formaldehyde- $\mathrm{CaCl}_{2}$ fixative (2\% paraformaldehyde, $1 \%$ $\mathrm{CaCl}_{2}$ ) in $0.1 \mathrm{M}$ cacodylate buffer containing $7 \%$ sucrose for $4 \mathrm{~h}$ at $4^{\circ} \mathrm{C}$. For whole-mount preparations, the tissues were separated into several layers under a dissection microscope by carefully pulling them apart with watchmaker's forceps. At least five tissue strips of each layer were obtained from each jejunum and kept in $0.1 \mathrm{M}$ cacodylate buffer containing $7 \%$ sucrose. In addition, several pieces of the fixed jejunum were placed in $0.1 \mathrm{M}$ cacodylate buffer containing $7 \%$ sucrose for at least $24 \mathrm{~h}$ at $4^{\circ} \mathrm{C}$ and embedded in OCTcompound (Miles, Diagnostic Division, Elkhart, IN, USA). Then, longitudinal sections were cut at a $7 \mu \mathrm{m}$ thickness with a cryostat and mounted on a glass slide. These tissue preparations were processed for enzyme-histochemical staining for $5^{\prime}$-nucleotidase (5'-Nase) or alkaline phosphatase (ALPase) activity.

\section{Enzyme-histochemistry}

The tissue preparations were stained with $5^{\prime}$-Nase staining or $5^{\prime}$-Nase-ALPase double staining as follows (Kato and Miyauchi, 1989; KATO, 1990a, b; KATO and GoTOH, 1990; KATO et al., 1991, 1993). The specimens were washed with $0.1 \mathrm{M}$ cacodylate buffer (pH 7.2) containing 7\% sucrose and incubated in the standard medium for $5^{\prime}$-Nase activity for $45 \mathrm{~min}$ at $37^{\circ} \mathrm{C}$ with $5^{\prime}$-adenosine monophosphate (AMP) (Sigma Chemical, St. Louis, MO, USA) as the substrate and lead nitrate (TAAB Laboratories Equipment, Berkshire, England) as the capture agent. Two $\mathrm{mM} \mathrm{L-}$ tetramisole (Sigma) as an inhibitor of nonspecific ALPase was added to the standard medium to obtain the specific reaction product for $5^{\prime}$-Nase activity. After washing with distilled water, the tissues were treated with $1 \%$ ammonium sulfide solution for $2 \mathrm{~min}$ at room temperature. For 5'-Nase-ALPase double staining, they were then treated for $30 \mathrm{~min}$ at $4^{\circ} \mathrm{C}$ with the reaction medium for ALPase activity according to the azo-dye method with naphthol AS-MX phosphate (disodium salt) (Sigma) as the substrate and Fast blue BB (Sigma) as the coloring agent. Control stainings for the reactions were performed as follows: 1) omission of the substrate (AMP or naphthol AS-MX phosphate) from the reaction medium; 2) addition $50 \mathrm{mM} \mathrm{NiCl}$ or $5 \mathrm{mM}$ L-tetramisole into the reaction medium; 3 ) incubation of the preparations for $60 \mathrm{~min}$ at $60^{\circ} \mathrm{C}$ before treatment with the reaction medium. All specimens were mounted on glass slides with Crystal/mount (biomeda, Foster, CA, USA) and examined with a dissecting microscope (Stemi SV II) (Zeiss, Oberkochen, Germany) and a light microscope (Olympus BH-2, Tokyo, Japan).

\section{Scanning electron microscopy (SEM)}

After staining for $5^{\prime}$-Nase activity, several wholemount preparations were dehydrated in a graded ethanol series and dried by the t-buthylalcohol freezedrying method (InOUÉ and OsATAKe, 1988). The dried specimens were mounted on an aluminium stub with double-sided tape, and then uniformly coated with carbon for backscattered electron imaging, followed by examination with a scanning electron microscope (SEM) (S-800, Hitachi) equipped with a backscattered electron detector. The backscattered electron micrographs were taken as reported previously (KATO, 1990b; KATO and GOTOH, 1990).

\section{RESULTS}

\section{Enzyme reaction of lymphatic vessels}

Those lymphatic vessels showing positive staining for $5^{\prime}$-Nase activity revealed the lead sulfide reaction product colored a dark brown in the whole-mount preparations as well as tissue sections of the monkey jejunum under a light microscope (Fig. 1a). In contrast, significantly lower or no $5^{\prime}$-Nase activity at all was recognized in the blood vessels. Lymphatics with $5^{\prime}$-Nase activity were demonstrated in all layers of the jejunal wall including the lamina muscularis mucosae (Fig. 2a). After staining for ALPase, the blue reaction product was restricted to blood vessels, the capillaries and arterioles particularly, clearly contrasting with the dark brown lymphatic vessels (Fig. 1b). The mucosal epithelium and smooth muscle were also stained with ALPase staining (Fig. 2a). No remarkable changes in the intensity and localization of the reaction product of $5^{\prime}$-Nase activity after $5^{\prime}$ Nase-ALPase double staining were observed as compared with those after $5^{\prime}$-Nase staining alone (Fig. 1). The control stainings for $5^{\prime}$-Nase and ALPase activity gave completely negative results. 


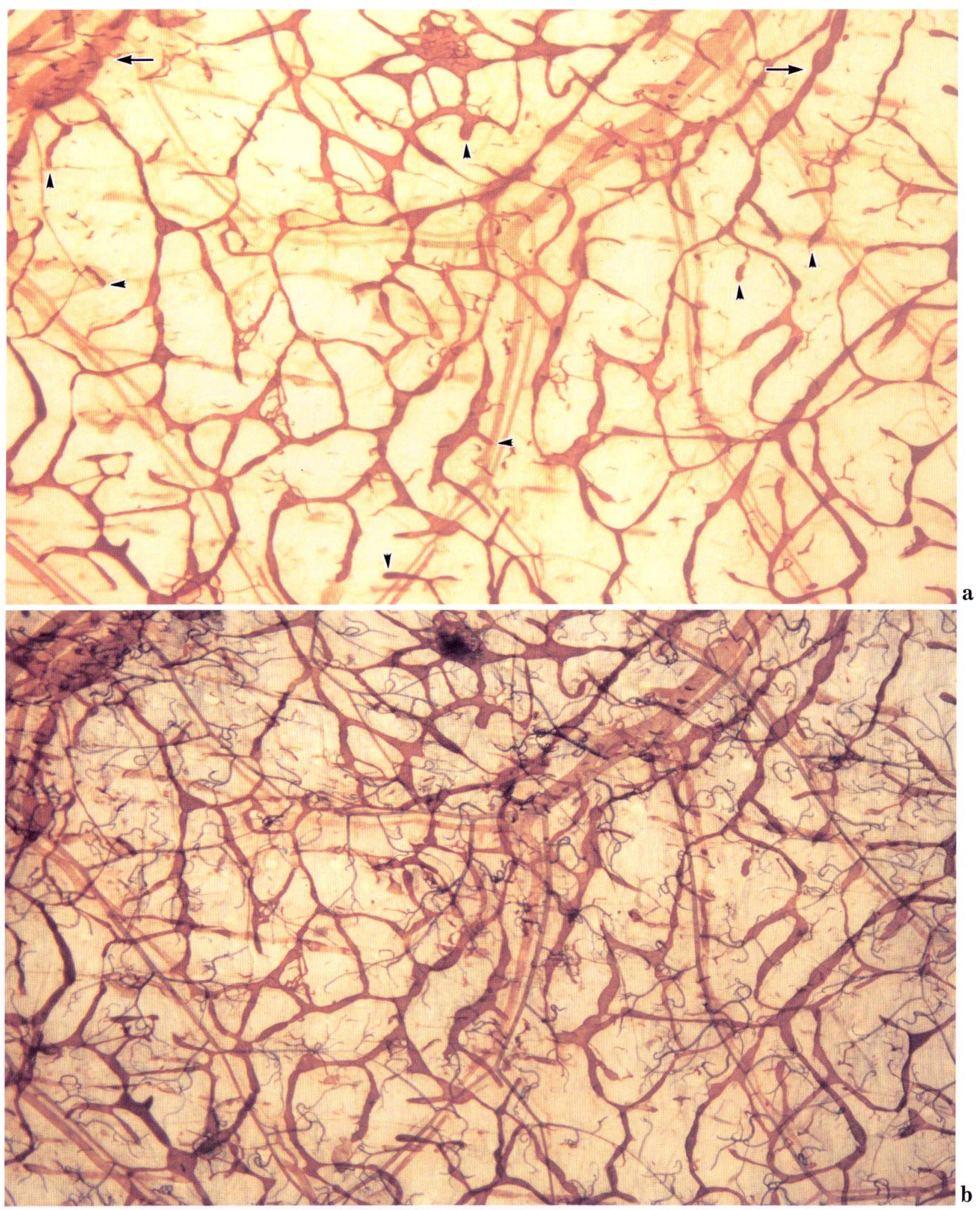

Fig. 1. Light micrographs of a whole-mount preparation of the submucosal layer of the monkey jejunum with histochemical demonstration of lymphatics and blood vessels. a. 5'-Nase activity. A dense network of lymphatic vessels (dark brown) with intense $5^{\prime}$-Nase activity is shown throughout the submucosal layer. Blood vessels reveal lower or no activity. The knotty structures of the large lymphatics are indicated with arrows. The knob-like endings of the initial lymphatics are indicated with arrowheads. b. 5'-Nase and ALPase activity of the same preparation followed by ALPase staining. A network of blood vessels (blue) is demonstrated per ALPase activity. a, b: $\times 10$ 

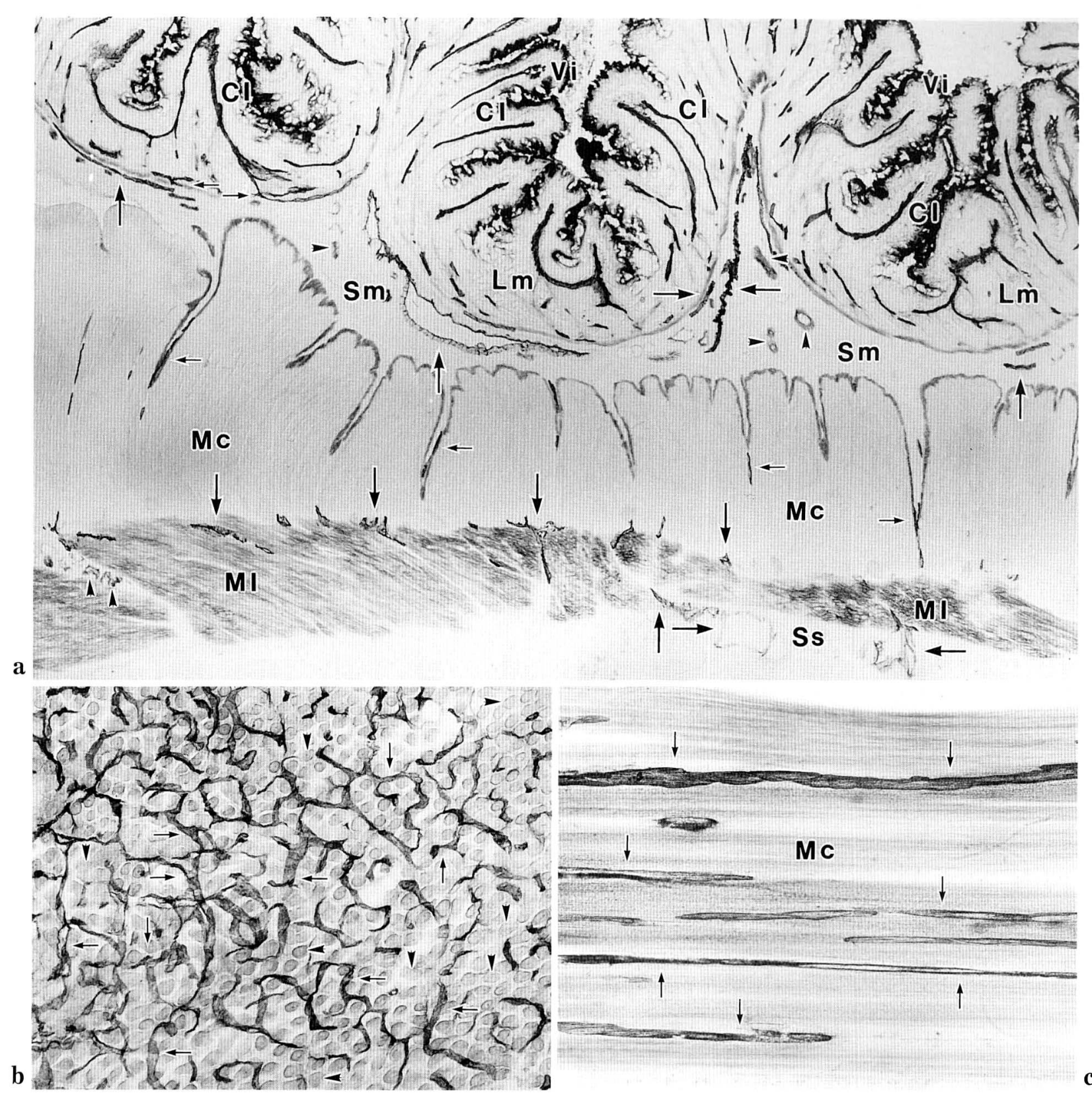

Fig. 2. Light micrographs of a longitudinal cryostat section (a) and whole-mount preparations (b, c) of the jejunum with histochemical staining for demonstration of the lymphatics and blood vessels. a. 5'-Nase-ALPase double staining. Lymphatics (arrows) react positively for 5'-Nase activity, whereas blood vessels (arrowheads) are positive for ALPase activity. The slender lymphatics (small arrows) running perpendicularly through the muscularis mucosae and the circular muscle layer, are also observed. $\mathrm{Vi}$ villi, $\mathrm{Cl}$ central lymphatic vessel, $\mathrm{Lm}$ lamina propria, $S m$ submucosa, $M c$ circular muscle, $M l$ longitudinal muscle, Ss subserosa. $\times 50$. b. $5^{\prime}-$ Nase staining of the basal part of the jejunal mucosa. A irregular network consisting of $5^{\prime}$-Nase-positive lymphatics (arrows) is demonstrated around the intestinal glands (arrowheads). $\times 35$. c. $5^{\prime}$-Nase staining of circular muscle layer $(M c)$, showing tubular lymphatic vessels (arrows). $\times 65$ 

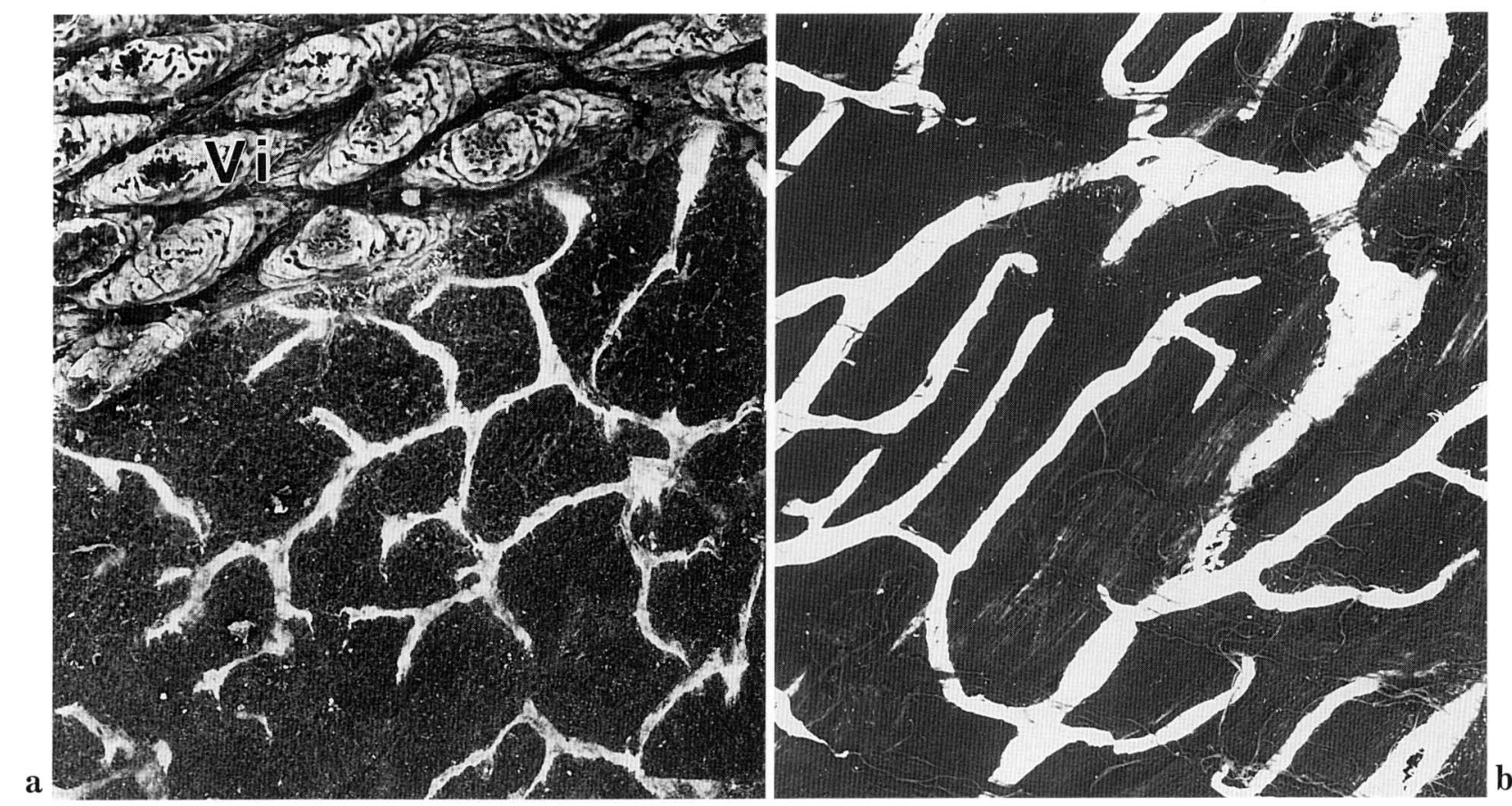

Fig. 3. Backscattered imaging-scanning electron micrographs of the whole-mount preparations of mucosal (a) and myenteric (b) layers stained with $5^{\prime}$-Nase staining. The $5^{\prime}$-Nase- positive lymphatics are strongly highlighted, corresponding to the light microscopic profiles shown in Figures $2 \mathrm{~b}$ and $4 \mathrm{a}$, respectively. $V i$ villi. a: $\times 70$, b: $\times 50$

\section{Distribution of lymphatic vessels}

The lymphatics, as demonstrated by $5^{\prime}$-Nase staining, extended as central lymphatic vessels all the way to the surface in the lamina propria of each villus (central lacteals). In other layers including the basal region of the mucosa, $5^{\prime}$-Nase-positive lymphatics appeared as irregularly-shaped spaces (Fig. 2a).

The rod-shaped central lacteals, which measured $300-600 \mu \mathrm{m}$ in height and $10-40 \mu \mathrm{m}$ in diameter, were slightly larger in diameter at the upper one fourth than those at the lower three fourths. Most lymphatics in the villi were several times larger in caliber than the blood capillaries.

Mucosal layer: The tissue strips of the jejunum containing the mucosal layer, from which the intestinal villi were removed, were stained with $5^{\prime}$-Nase staining. In the basal region of the jejunal mucosa, tubular shaped (10-60 $\mu \mathrm{m}$ in caliber) lymphatic vessels with $5^{\prime}$-Nase activity were distributed around the intestinal glands and formed an irregular network (Fig. 2b). This network was continuous with both the central lacteals and the slender connective tubules in the muscularis mucosae, which ran downward to drain into the submucosal lymphatics (Fig. 2a).

The backscattered electron imaging of a whole- mount preparation including the mucosal layer stained for $5^{\prime}$-Nase activity is illustrated in Figure 3a. Reaction products for $5^{\prime}$-Nase activity with deposits of heavy metal (lead) can be observed as strong highlights in the backscatter images (Fig. 3a), corresponding to the light microscopic profiles tinged dark brown (Fig. 2b). The same mucosal lymphatic network as that observed under the light microscope is clearly visualized.

Submucosal layer: The tissue preparations of each jejunum containing the submucosal layer were stained with $5^{\prime}$-Nase staining. Numerous lymphatic vessels with $5^{\prime}$-Nase activity were distributed throughout the submucosal layer (Fig. 1a). A network with polygonal meshes consisting of tubulo-saccular lymphatics of various thicknesses $(30-200 \mu \mathrm{m}$ in caliber) spread horizontally closely beneath the muscularis mucosae (Fig. 1a). The large lymphatics (approximately $300 \mu \mathrm{m}$ in caliber), which showed repeated knotty structures indicative of the locations of valves, ran parallel with large blood vessels between the mesh-like lymphatic network and circular muscle layer to the mesenteric border, branching at different sites along the course of the vessels (Fig. 1). The branches of these lymphatics formed a network as mentioned above and frequently revealed a 


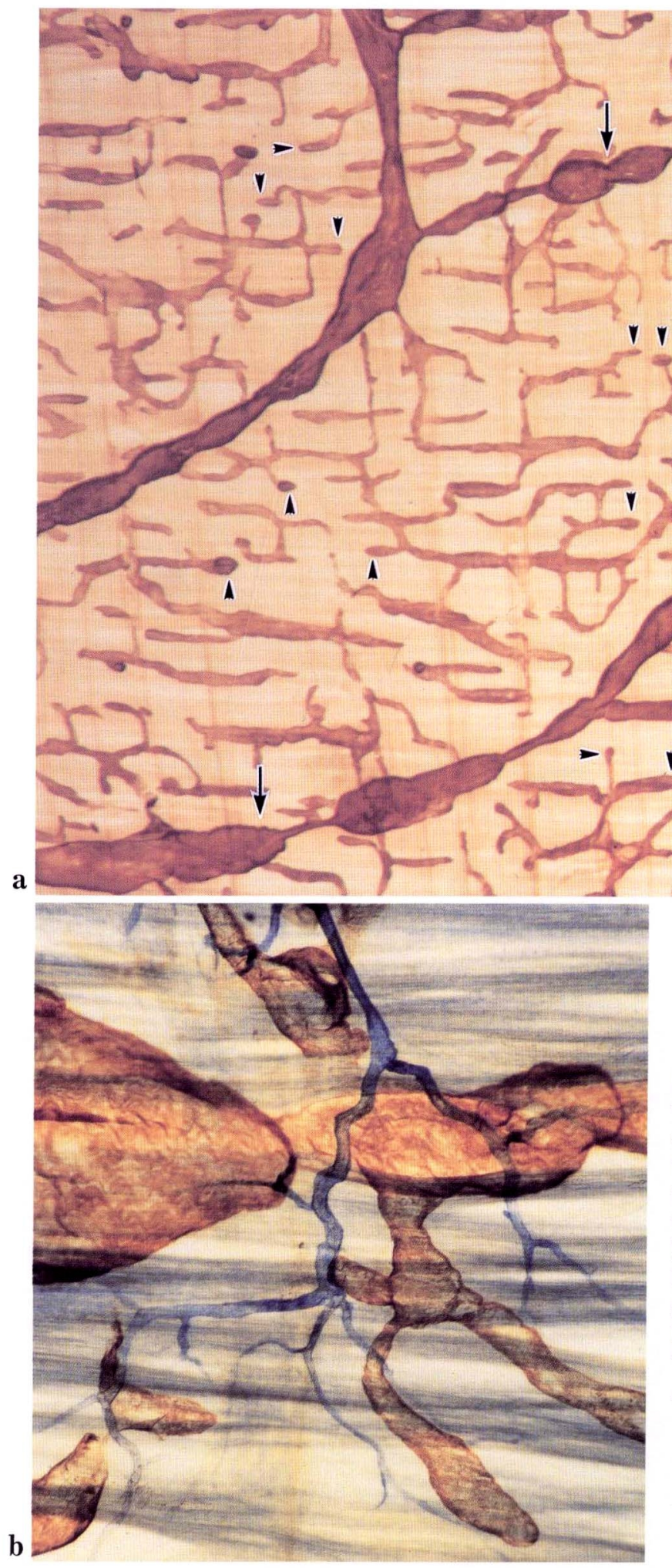

Fig. 4. Light micrographs of the whole-mount preparations of the myenteric layer with histochemical staining. a. $5^{\prime}$-Nase staining. A fine maze-like network of lymphatic vessels with intense $5^{\prime}$-Nase activity is demonstrated throughout the myenteric layer. The knotty structures of the large lymphatics are indicated with arrows. Marked knob-like endings are seen at the initial parts of the lymphatics (arrowheads). $\times 15$. b. $5^{\prime}$-Nase-ALPase double staining of other material including the myenteric layer. Blue blood vessels with ALPase activity are also seen. The smooth muscle layer is also colored blue due to its ALPase activity. $\times 90$ 


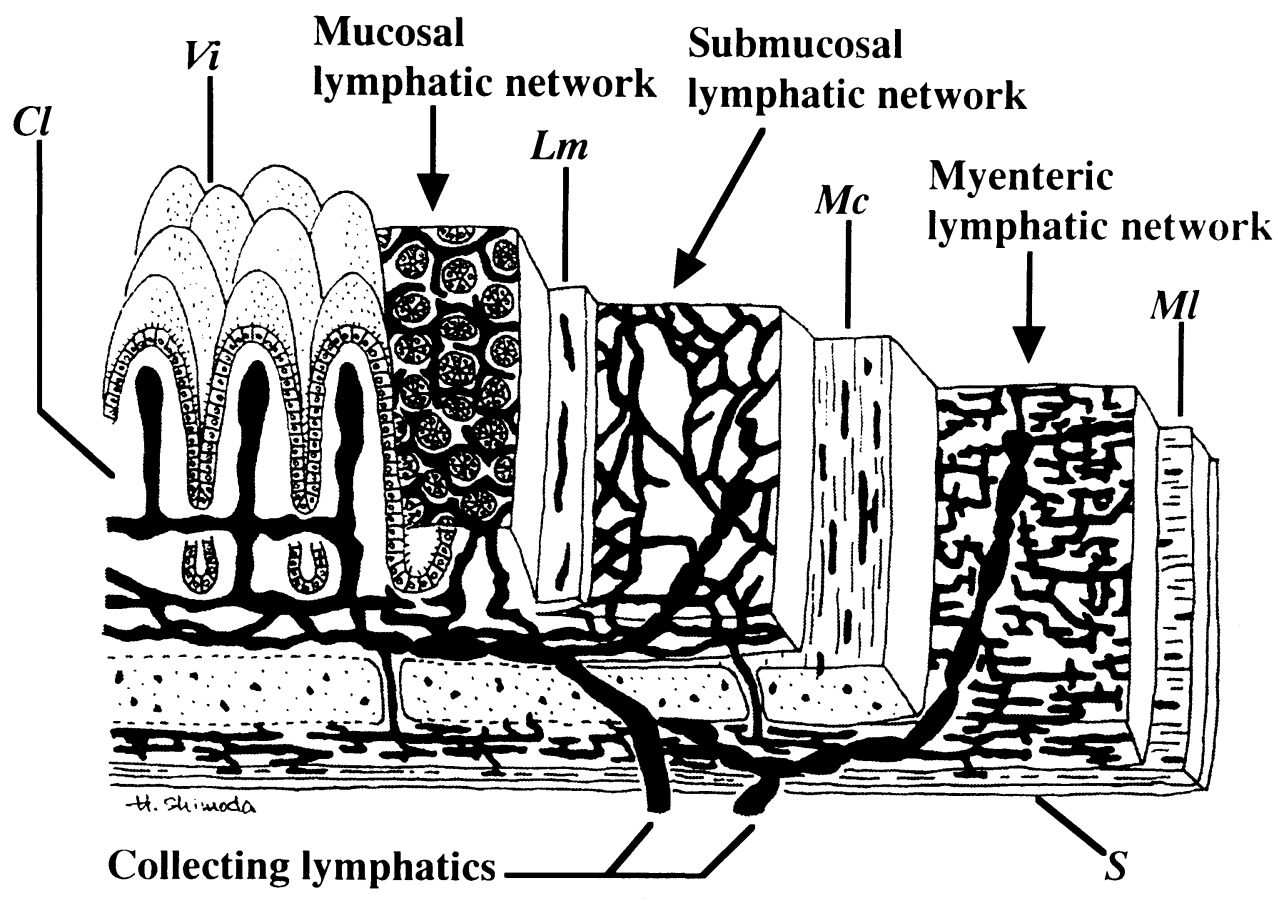

Fig. 5. A schematic presentation of the lymphatic system in the monkey jejunal wall based on the enzyme-histochemical study. $V i$ villi, $C l$ central lymphatic vessel, $L m$ lamina muscularis mucosae, $M c$ circular muscle layer, $M l$ longitudinal muscle layer, $S$ serosa.

varicose profile (Fig. 1). The apical portions of the initial lymphatics demonstrated many knob-like blind endings (Fig. 1a). The submucosal lymphatic network formed connections with the mucosal network by slender lymphatic vessels (5-10 $\mu \mathrm{m}$ in caliber) passing perpendicularly or obliquely through the lamina muscularis mucosae (Fig. 2a). This lymphatic network was predominantly independent of the network of blood capillaries, although the large lymphatics and some segments of the initial lymphatics were accompanied by blood vessels (Fig. 1b).

Circular muscle layer: The circular muscle coat contained sparse $5^{\prime}$-Nase-positive tubular shaped lymphatic vessels $(10-50 \mu \mathrm{m}$ in caliber) in all five strips of each monkey jejunum containing circular muscle (Fig. 2c). They ran along the smooth muscle bundles in the muscle coat, though a distinct network formation was not observed in this layer. In cryostat sections with $5^{\prime}$-Nase staining, slender lymphatic vessels (5-10 $\mu \mathrm{m}$ in caliber) which traveled downward without ramification were also demonstrated (Fig. 2a).

Myenteric layer, longitudinal muscle and serosa: Numerous $5^{\prime}$-Nase-positive lymphatic vessels of various thicknesses (40-200 $\mu \mathrm{m}$ in caliber) were distributed throughout the myenteric area, longitudinal muscle and serosa (Fig. 4a). The lymphatics with $5^{\prime}$-Nase activity in the myenteric area formed a well-developed network characterized by a maze-like distribution in a single layer (Fig. 4a). Each initial lymphatic vessel, which showed this maze-like distribution, branched from different sites of the large lymphatic vessels (approximately $300 \mu \mathrm{m}$ in caliber) along their course and appeared tubulo-saccular in shape (Fig. 4a). Numerous knob-like blind endings were observed at the apical parts of these initial lymphatics (Fig. 4a). These lymphatic vessels ran predominantly along the muscle bundles and drained into the large lymphatics at various sites (Fig 4a). The large lymphatics in the myenteric layer, with marked knotty structures indicative of the locations of valves, ran into the subserosal layer by obliquely penetrating the longitudinal muscle layer at the mesenteric border (Figs. 2a, 4a) and drained into the mesenteric collecting lymphatics as described in our previous report (KATO et al., 1993). Under $5^{\prime}$-NaseALPase double staining, some segments of the lymphatics were shown to be accompanied in part by 
blood vessels, although the network of initial lymphatics was mostly independent of the network of blood capillaries (Fig. 4b).

Figure $3 \mathrm{~b}$ shows the backscattered electron imaging of a whole-mount preparation including the myenteric layer after $5^{\prime}$-Nase staining. Such a $5^{\prime}$ Nase-positive lymphatic network revealed strong highlights in backscatter images. In the longitudinal muscle layer, the tubular lymphatics $(10-50 \mu \mathrm{m}$ in caliber) with $5^{\prime}$-Nase activity were sparsely distributed along the muscle bundles.

\section{DISCUSSION}

The present histochemical study using $5^{\prime}$-Nase-ALPase double staining enabled a differentiation between $5^{\prime}$-Nase-positive lymphatics and ALPase-positive blood vessels in whole-mount preparations of the monkey jejunal wall. The intensity and localization of the $5^{\prime}$-Nase and ALPase reactions in the walls of the lymphatics and blood vessels under discussion resemble those observed in our previous studies (KATO et al., 1991, 1993). Thus, application of this method to whole-mount preparations of the laminar layers serves in demonstrating the lymphatic system in the intestinal wall.

The central lacteals ending blind in the villi were rod-like in appearance in the monkey jejunum, corresponding to previous SEM observations using corrosion cast techniques in other mammals (rabbit: OHTANI and OHTSUKA, 1985; rat: OHTANI, 1987; canine: YAMANAKA et al., 1995). The lacteals anastomosed to each other at the villous base to form an irregular network which horizontally extended around the intestinal glands. This mucosal lymphatic network likely acts as a reservoir for fluid transport from and/or into the central lymphatic vessels in the villi, as OHTANi and OHTSUKa (1985) noted in their study in rabbit intestine. In the muscularis mucosae, slender lymphatics without ramification were observed connecting the mucosal lymphatic network with the submucosal network; they presumably contribute a constant flow of lymph from the mucosal lymphatic reservoir into the submucosal lymphatics.

In the submucosal layer of the monkey jejunum, a network of tubulo-saccular lymphatic vessels extended horizontally beneath the lamina muscularis mucosae. In their corrosion casting/SEM study, YAMANAKA et al. (1995) showed two kinds of lymphatic networks located beneath the lamina muscularis mucosae and above the circular muscle layer in the submucosal layer of the canine small intestine. In the present study, however, the submucosal layer contained only a single lymphatic network. Previous SEM studies of lymphatic corrosion casts in the rabbit and rat small intestine (OHTANI and OHTSUKA, 1985; OHTANI, 1987) report a single submucosal network of lymphatics similar to that observed in this study. This discrepancy may be due to the species difference.

The large lymphatics with repeated knotty structures indicative of the locations of valves were observed running parallel with large blood vessels to the mesenteric border in the submucosal layer as well as the myenteric layer of the intestine. Since the morphological features of these large lymphatics correspond to those of the collecting lymphatics described in previous reports including SEM studies (OHTANI and OHTSUKa, 1985; OHTANI, 1987, 1992; OHTANI and MuraKami, 1987; KATO et al., 1993; YAMANAKA, 1995), they are considered to be collecting tubules.

In contrast, the branches of the large lymphatics in the submucosal layer, which supplied the network, are regarded as the initial lymphatics (lymphatic capillaries). In addition, these lymphatics have frequently displayed varicose structures not observed in our previous study of the rat small intestine (SHIMODA et al., 1996). Several studies have found that some initial lymphatics possess distinct valve structures (HoGAN and UNTHANK, 1986a, b; MAZZONI et al., 1987; KATO et al., 1993; OHTANI et al., 1993). Despite the need for further studies as to whether the varicose structures of the initial lymphatics represent the locations of the valves, these structures are believed to prevent the reflux of lymph flow in the initial lymphatics. They may also play a part in protecting the excessive dilation and/or oppression of the lymphatic wall. The lymphatic capillaries with knob-like blind endings in the submucosal layer corresponded morphologically to those in previous studies using the microinjection of dyes and SEM techniques (ZWEIFACH, 1973; OHTANI, 1992). Therefore, the present findings suggest that the tissue fluid in the submucosa is actively drained into the initial lymphatic network and transported through the submucosal collecting tubules into the mesenteric lymphatics (KATo et al., 1993).

In the circular and longitudinal muscle layers, the tubular lymphatics with blind endings, which appeared to connect predominantly with the myenteric lymphatic network, were sparsely distributed along the muscle bundles. In contrast, the slender lymphatics, judged to be connecting tubules between the submucosal lymphatic network and the myenteric network, were also observed passing perpendicularly through the circular muscle layer; they likely serve in the flow of lymph between submucosal and myenteric 
lymphatic networks.

In the myenteric layer, $5^{\prime}$-Nase-positive thick lymphatics of a well-developed network appeared like collecting lymphatics with valves, and the other lymphatics joining those thick lymphatics at various sites are considered to be initial lymphatics. In this layer, numerous apical parts of the initial lymphatics revealed marked knob-like blind endings like those in the submucosal layer. These findings imply that the tissue fluid in the muscular and myenteric layers is actively absorbed into the lymphatic capillaries and transported into the mesenteric lymphatics through the collecting tubules.

The overall structural organization of the lymphatic system in the monkey jejunum revealed by our histochemical method is schematically presented in Figure 5 . The distribution of the initial lymphatics in the monkey intestinal wall was denser than that in the rat small intestinal wall; similar lymphatic networks were seen in the rat intestine in our previous study (SHIMODA et al., 1996). The lymphatic networks presumably act as a reservoir for fluid transport, since they showed relatively horizontal spreading. They further may prevent lymph stasis caused by contractive movements of the intestinal wall such as peristalsis. The initial lymphatic networks in the monkey jejunum are independent of the networks of blood vessels with ALPase activity, except for that the blood vessels run in proximity with certain segments of the lymphatic vessels. OHTANI and OHTSUKA (1985) suggested that blood vascular movement represents an important energy source aiding lymph formation and transport in the rabbit small intestine. Therefore, in the monkey intestinal wall as well, vasomotion may partly affect lymph formation and transport. These possibilities remain to be examined in future studies.

Acknowledgements. We thank Professor T. Usu for his valuable cooperation, and Mr. T. KAJIWARA for his expert technical assistance.

\section{REFERENCES}

Allen, L.: Lymphatics and lymphoid tissue. Ann. Rev. Physiol. 29: 197-224 (1967).

Hogan, R. D. and J. L. UNThANK: Mechanical control of initial lymphatic contractile behavior in bat's wing. Amer. J. Physiol. 251: H357-363 (1986a).

- - The initial lymphatics as sensors of interstitial fluid volume. Microvasc. Res. 31: 317-324 (1986b).
InOUE, T. and H. Osatake: A new drying method of biological specimens for scanning electron microscopy: The t-buthyl alcohol freeze-drying method. Arch. Histol. Cytol. 51: 53-59 (1988).

KATo, S.: Enzyme-histochemical demonstration of intralobular lymphatic vessels in the mouse thymus. Arch. Histol. Cytol. 53, Suppl.: 87-94 (1990a).

- Enzyme-histochemical identification of lymphatic vessels by light and backscattered image scanning electron microscopy. Stain Technol. 65: 131-137 (1990b).

KATo, S. and M. Gотон: Application of backscattered electron imaging to enzyme histochemistry of lymphatic capillaries. J. Electron Microsc. 39: 186-190 (1990).

KATo, S. and R. MiYAUCHI: Enzyme-histochemical visualization of lymphatic capillaries in the mouse tongue: Light and electron microscopic study. Okajimas Fol. Anat. Jap. 65: 391-404 (1989).

Kato, S., A. Yasunaga and Y. Uchida: Enzyme-histochemical method for identification of lymphatic capillaries. Lymphology 24: 125-129 (1991).

Kato, S., M. MiURA and R. MiYaUChi: Structural organization of the initial lymphatics in the monkey mesentery and intestinal wall as revealed by an enzymehistochemical method. Arch. Histol. Cytol. 56: 149-160 (1993).

Mazzoni, M. C., T. C. SkalaK and G. W. SchmidshonBEIN : Structure of lymphatic valves in the spinotrapezius muscle of the rat. Blood Vessels 24: 304-312 (1987).

MoRI, K.: Identification of lymphatic vessels after intraarterial injection of dyes and other substances. Microvasc. Res. 1: 268-274 (1969).

OHTANI, 0.: Three-dimensional organization of lymphatics and its relationship to blood vessels in rat small intestine. Cell Tiss. Res. 248: 365-374 (1987).

- Structure of lymphatics in rat cecum with special reference to submucosal collecting lymphatics endowed with smooth muscle cells and valves. I. A scanning electron microscopic study. Arch. Histol. Cytol. 55: 429-436 (1992).

Ohtani, 0. and T. Murakami: Lymphatics and myenteric plexus in the muscular coat in the rat stomach: A scanning electron microscopic study of corrosion casts made by intra-arterial injection. Arch. Histol. Jap. 50: 87-93 (1987).

Ohtani, O. and A. OHTSUKa: Three-dimensional organization of lymphatics and their relationship to blood vessels in rabbit small intestine. A scanning electron microscopic study of corrosion casts. Arch. Histol. Jap. 48: 255-268 (1985).

Ohtani,Y., O. Ohtani and T. Nakatani: Microanatomy of the rat diaphragm: A scanning electron and confocal laser scanning microscopic study. Arch. Histol. Cytol. 56: 317-328 (1993).

Satomura, K., N. Tanigawa and S. Magari: Microlymphangiographic study of lymphatic regeneration following intestinal anastomosis. Surg. Gynecol. Obstet. 146: 415-418 (1978). 
Shimoda, H., S. Kato, T. Kudo and T. Usui: Enzymehistochemical demonstration of lymphatic network in the rat small intestinal wall (In Japanese with English summary). Jap. J. Lymphol. 19: 65-71 (1996).

Sugito, M., K. Araki and T. Ogata: Three-dimensional organization of lymphatics in the dog stomach: A scanning electron microscopic study of corrosion casts. Arch. Histol. Cytol. 59: 61-70 (1996).

Usнiki, T.: The three-dimensional organization and ultrastructure of lymphatics in the rat intestinal mucosa as revealed by scanning electron microscopy after KOH-collagenase treatment. Arch. Histol. Cytol. 53, Suppl.: 127-136 (1990).

VAJDA, J. and T. TömвöL: Die Lymphgefäßstruktur der Dünndarmwand. Acta Morphol. Acad. Sci. Hung. 13: 339-347 (1964).

Yamanaka, Y., K. Araki and T. Ogata: Three-dimensional organization of lymphatics in the dog small intestine: A scanning electron microscopic study on corrosion casts. Arch. Histol. Cytol. 58: 465-474 (1995).

Yasunaga, A., S. Kato, Y. Uchida and R. MiYauchi: Enzyme-histochemical study on the fine distribution of the intramural lymphatics at the ileocecal junction of the monkey intestine. Okajimas Fol. Anat. Jap. 68: 259270 (1991a).
Yasunaga, A., Y. UChida and S. Kato: Enzyme-histochemical study of lymphatics and blood vessels in the lamina propria mucosae of the human large intestine (In Japanese with English summary). Jap. J. Lymphol. 14: 13-17 (1991b).

ZWEIFACH, B. W.: The microcirculation in the intestinal mesentery. Microvasc. Res. 5: 363-367 (1973).

Dr. Hiroshi SHIModA Department of Anatomy Oita Medical University 1-1 Idaigaoka, Hasama-machi Oita, 879-55 Japan

下田浩 879-55 大分県大分郡挾間町医大ヶ 丘 1-1 大分医科大学

解剖学講座 